\title{
Tibial spurs (pes anserinus bony spurs) in children and adolescents: a case series of 21 patients
}

\section{Tomoya Matsunobu ( $\square$ matsunob@ortho.med.kyushu-u.ac.jp )}

Kyushu Rosai Hospital https://orcid.org/0000-0002-5431-4975

\section{Akira Maekawa}

Kyushu Rosai Hospital

\section{Suguru Fukushima}

Kyushu Rosai Hospital

\section{Akira Nabeshima \\ Kyushu Rosai Hospital \\ Yukihide Iwamoto \\ Kyushu Rosai Hospital}

\section{Research article}

Keywords: Exostosis, tibial spur, pes anserinus bony spur, pes anserinus bursitis, osteochondroma

Posted Date: August 19th, 2020

DOI: https://doi.org/10.21203/rs.3.rs-43646/v2

License: @ (i) This work is licensed under a Creative Commons Attribution 4.0 International License. Read Full License 


\section{Abstract}

Background: Icicle-like bony spurs that originate from the proximal medial surface of the tibia in young persons have been referred to by various names in the literature. This tibial spur, called pes anserinus bony spurs (PABSs), can present as exostoses, but lacks a cartilage cap which is characteristic feature of osteochondroma. To date, there are only two small case series about the tibial spur, and little information is available. The aim of this study was to describe the characteristics of PABSs in children and adolescents.

Methods: This retrospective study analysed patients diagnosed with PABSs on an orthopaedic oncology service from 2013 to 2020. Additionally, in order to estimate the prevalence of PABSs in children and adolescents, we retrospectively reviewed knee X-rays of patients who visited our hospital for other reasons from 2008 to 2020.

Results: Thirteen male and 8 female patients with PABSs, with a mean age of 11.7 years, were evaluated. Bilateral spurs were observed in 7 of the 21 patients. Among 28 PABSs in 21 patients, 6 were incidentally identified. Radiological evaluation showed that the average PABS length was $10.6 \mathrm{~mm}$. There was a statistically significant difference in the spur length between incidentally detected PABSs and symptomatic PABSs at the first visit. The prevalence rate of PABSs among children and adolescents was $0.94 \%$. Computed tomography examinations showed that no PABSs occurred at the site of the tendinous insertion of the pes anserinus. Surgical resection was performed in only 2 patients, both of whom were high-performing athletes.

Conclusions: This is the first study to describe the clinical and radiological features of PABSs in detail and to report their prevalence in children and adolescents. Although longer PABSs are symptomatic, most PABSs are non-problematic and surgical resection of PABSs should be considered only if pain persists despite conservative treatment.

\section{Background}

Osteochondroma is the most common bone neoplasm, and takes the form of bony outgrowth on the surface of the bone. If we are only taking about the proximal medial surface of the tibia in young persons, however, bony outgrowth is not always osteochondroma. Ugai et al. published the first and largest case series of ten patients with bony spurs on the pes anserinus (1). These spurs had an icicle-like shape, and surgically resected spurs were not covered with a cartilage cap. The authors proposed that icicle-like spurs without a cartilage cap could be classified as pes anserinus bony spurs (PABSs). Thus far, only 2 small case series of PABSs in young patients have been reported $(1,2)$. Little information is available on the relationship between the radiographic characteristics of PABSs and symptoms such as pain. Furthermore, the etiology of PABSs remains unclear. To characterize PABSs in children and adolescents, in this retrospective study we reviewed a series of 21 young patients who were referred to a specialist orthopaedic oncology service with a presumptive diagnosis of a bone tumour on the medial side of the proximal tibial metaphysis, but who were ultimately shown to have PABSs. Additionally, we assessed the prevalence of PABSs in children and adolescents.

\section{Patients And Methods}

This study was approved by the ethical committee of our hospital. In this retrospective study, patients with PABSs between January 2013 and March 2020 were retrieved from the database of the Orthopaedic Oncology 
Service, Department of Orthopaedic Surgery. We made a diagnosis as PABSs when bony spurs occurred on the medial side of the proximal tibial metaphysis, and morphologically were shaped like a rose thorn or icicle, with a sharp tip on plain AP X-rays (Fig. 1A). The length of PABSs was determined by measuring the distance from the tip of PABSs to the midpoint of the base of PABSs on an anteroposterior plain X-ray. The midpoint of the base of PABSs was defined as center of a line to connect the most proximal point of the contour of PABSs to the most distal point of that (Fig. 1A'). To determine the position of each PABS on a lateral plain X-ray, the distance from the anterior edge of the tibia to the base of the PABS was divided by the anteroposterior diameter parallel to the proximal tibial growth plate and level with the base of the spur (Fig. 1B and C). The follow-up duration ranged from 3 to 47 months (mean 20.0 months). We applied an unpaired t-test when comparing parametric data between groups.

To determine the prevalence of PABSs in children and adolescents, our hospital's electronic data warehouse was queried to identify young patients (ages 6-16) who underwent knee or lower leg X-ray examinations between January 2008 and March 2020 for various reasons (e.g., sprain, contusion, and fracture).

\section{Results}

Demographic and clinical characteristics of PABS cases are summarised in Table 1. There were 13 male and 8 female patients, and the age at the initial visit ranged from 6 to 16 years (mean 11.7 years). Medical interviews revealed that the duration from initial symptoms to the first hospital visit ranged from 1 day to 1 year (median 1 month). Except for 1 (case \#13) patient in whom a unilateral PABS was incidentally identified when she underwent X-ray examination of the lower leg for ankle sprain, all patients visited a primary care or orthopaedic clinic due to unilateral or bilateral gonalgia, and then they were referred to us with a presumptive diagnosis of bone tumour. Most patients complained of knee pain, especially during exercise, but none experienced pain at rest. Physical examination at our hospital revealed that 18 patients experienced knee pain during range of motion (ROM) exercises, and 17 reported tenderness over the affected medial proximal tibia.

In each patient, an X-ray of the unaffected side was obtained for contralateral comparison, even if that side was asymptomatic. This resulted in the unexpected identification of 5 PABSs. Overall, bilateral spurs were observed in 7 of the 21 patients, there were 28 PABSs in 21 patients, and 6 of 28 PABSs were incidentally identified (cases \#1, 2, 4, 8, 12 and 13). Radiographic analysis of AP images revealed that the mean PABS length was $10.6 \mathrm{~mm} \pm 5.2 \mathrm{~mm}$ (5 to $25 \mathrm{~mm}$ ) (Fig. 2A and Table 1). Radiographic analysis of lateral images revealed that PABSs were positioned slightly posterior to the medial edge of the proximal tibia (the distance from the anterior edge of tibia to the spur was $59.2 \% \pm 8.8 \%$ of the total distance between the anterior and posterior edges) (Fig. $1 \mathrm{~B}, 1 \mathrm{C}$ and $2 \mathrm{~B}$, Table 1).

Next, we evaluated the relationship between PABSs and patient characteristics. Spur length did not correlate with either patient age or height (data not shown). In addition, we found that incidental detection (i.e., asymptomatic spurs) was statistically associated with spur length (Fig. 3) but not with location (data not shown). Magnetic resonance imaging examination was performed in 9 patients with 11 PABSs, and a hyperintense T2-weighted signal, which is characteristically observed in the cartilage cap of osteochondroma, was not identified on the surface of any PABS (Table 1). Computed tomography (CT) examination was performed in 8 patients with 11 PABSs. The radiopacity of each PABS differed from that of the underlying bone 
marrow, and there was no continuity between PABS and marrow (Fig. 1D). Interestingly, none of PABSs occurred at the site of the tendinous insertion of the pes anserinus, and the sartorius tendon (and pes anserinus) ran just beneath the PABSs (Fig. 1E and 1F).

At the last follow-up, 19 of 21 patients required no surgical treatment (Table 1). Because 2 patients (cases \#9 and \#17) complained of persistent pain over the site of the PABS, surgical resection was performed 6 and 1.5 months after the patients' first visits, respectively. Both patients were high-performing junior athletes (tennis and volleyball players, respectively), and their performance was impaired by pain. Intraoperative findings showed that the pes anserinus was compressed by the PABS during knee extension, and this compression was relieved during knee flexion (Fig. 4A and 4B). Histological examination of both resected specimens showed that the tips of the PABS lesions contained mature bone trabeculae and fatty marrow without cartilage caps (Fig. 4C).

These findings suggest that smaller PABSs may be indolent and asymptomatic, and thus easily overlooked. To determine the prevalence of PABSs in children and adolescents, we retrospectively reviewed knee X-rays of patients who visited our hospital for reasons unrelated to PABSs. From January 2008 to March 2020, X-rays of 1477 knees in 961 children and adolescent patients were obtained (ages 6-16). The aforementioned 21 patients who were referred to our orthopaedic oncology service and diagnosed with PABSs were excluded from the prevalence analysis. As a result of this analysis, 10 new PABSs in 9 patients were identified (Fig. 5). Therefore, the prevalence rate of PABSs among children and adolescents was $0.94 \%$.

\section{Discussion}

Because bony spurs that originate from the proximal medial surface of the tibia in young persons have been referred to by various names in the literature (Table 2), it seems that awareness of PABSs among orthopaedic surgeons may be low. Caffey initially described an asymptomatic transitory exostosis of the proximal tibial metaphysis in a 6-year old boy, and plain X-rays in that case showed an icicle-shaped bony spur (3). Ugai et al. reported 14 exostoses without a cartilage cap located on the pes anserinus in 10 patients, which they referred to as PABSs (1). Fraser et al. reported 10 children with pes anserinus syndrome due to proximal tibial bone spurs (2). Tiwari et al. reported that 2 of 3 patients with pes anserinus syndrome had an unusual presentation of rose thorn-like bony spurs without cartilage caps (4). Keats and Anderson presented a case of bilateral tibial spurs (5). The findings in each of the above studies seem to represent the same lesion as the tibial bony spurs in this study (Table 2). As in the above reports, our study revealed that PABSs manifest in the first 2 decades of life with no sex predilection.

To the best of our knowledge, this study is the first to radiographically evaluate PABSs. While involvement is usually unilateral, bilateral occurrence is not rare (33.3\%). PABSs were very small, with a maximum length of 25 $\mathrm{mm}$, and were consistently located slightly posterior to the medial edge of the proximal tibia. Smaller PABSs were asymptomatic and incidentally identified. The prevalence rate of PABSs in young persons was estimated to be $0.94 \%$.

The causes of PABSs remain unclear. The question inevitably arises as to whether PABS is a variant of osteochondroma, which is the most common benign bone neoplasm. Both osteochondroma and PABS exhibit an abnormal bony projection from the surface of a bone. However, there are a couple of crucial differences 
between them. First, PABS histologically consists of lamellar bone trabeculae and fatty marrow without a hyaline cartilaginous cap (Fig. 4C) $(1,2)$ while osteochondroma consists of a cartilage cap and underlying bone (6). Second, in this study, PABS radiopacity differed from that of the underlying parent bone marrow, with no continuity between the two structures (Fig. 1D), while in osteochondroma the cortex and medullary cavity of the underlying bone were found to be continuous with the bony stalk and the centre of the lesion (6). Third, this study showed that the bases of the PABSs were consistently located at the medial-posterior edge of the proximal tibia, suggesting that PABSs may be non-neoplastic, site-dependent lesions (Fig. 1E and 2), while osteochondromas usually arise from the metaphysis of long bones and may grow in any direction (6). Nevertheless, we cannot rule out the possibility that loss of the cartilage cap due to continuous mechanical stress may result in PABS formation (1). Indeed, several studies have reported the spontaneous regression of osteochondromas $(7,8)$. Since EXT genes were found to play a role in osteochondroma formation (9), histological and biological research of these genes may help to distinguish between PABS and osteochondroma.

Ugai et al. described another possible aetiology of these bony spurs, namely enthesopathic alteration of the pes anserinus tendon (1). Enthesopathy refers to a disorder at the enthesis, and repetitive trauma at the enthesis leads to a repair process that includes development of painful bony outgrowths called "traction spurs" (10). However, our 3D-reconstructed, volume-rendered CT images of soft tissue in this study clearly showed that PABSs did not occur at the site of the tendinous insertion of the pes anserinus, indicating that PABSs are unlikely to result from enthesopathy. Taking into consideration our intraoperative findings that PABSs collided with the pes anserinus, we suspect that pain caused by PABSs may not result from enthesopathy. Further histological and biological research is warranted to elucidate the exact mechanism of PABS formation.

Regarding the treatment of PABSs, surgical resection was performed in all patients by Ugai et al. and in half of patients by Fraiser et al. $(1,2)$. These patients may have been overtreated, because we revealed in this study that about a quarter of PABS cases were asymptomatic, and only 2 patients underwent surgical resection of PABS during the follow-up period. Tiwari et al. recommended conservative management for most patients with tibial spurs, with surgical treatment needed only rarely in recalcitrant cases (4). Caffey reported a 6-year old boy with an asymptomatic spur that disappeared after 3-4 years (3). These and other reports suggest that clinicians should first consider conservative treatments such as rest, and should perform surgical interventions only if conservative approaches have failed. Based on not only radiographic findings that PABSs were positioned slightly posterior to the medial edge of the proximal tibia and the pes anserinus ran just beneath the PABSs, but also intraoperative findings that the pes anserinus was compressed by the PABS during knee extension, surgical resection of PABSs should be considered in young athletes with repetitive knee flexion and extension only if pain persists despite conservative treatment.

A limitation of our study is the relatively short mean follow-up time of 20.0 months, as it is still unclear if PABSs eventually disappear over time. We have not identified any previous reports of symptomatic icicle-like tibial spurs in adulthood. As a result, we speculate that PABSs in children and adolescents may eventually become asymptomatic even if they last for many years.

In summary, we present the clinicopathologic and radiological features and outcomes of 21 patients with PABSs. The prevalence rate of PABSs among children and adolescents was $0.94 \%$. PABSs can be easily recognised because of their characteristic shape and location. Although larger PABSs may cause pes anserinus 
bursitis and pain, most PABSs are non-problematic. We suggest that surgical resection of PABSs should be considered in high-performing athletes only if pain persists despite conservative treatment.

\section{Abbreviations}

PABS: pes anserinus bony spur; CT Computed tomography

\section{Declarations}

Ethics approval and consent to participate

We obtained verbal informed consent from the patients' parents to use their medical data for publication. This study was a retrospective case-series study, carried out by the opt-out method approved by the ethics committee of Kyushu Rosai Hospital (18-24).

Consent for publication

Consent for publication in the study was obtained.

Availability of data and materials

The datasets used and/or analysed during the current study are available from the corresponding author on reasonable request.

Competing interests

The authors declare that they have no competing interests.

\section{Funding}

This work was in part supported by the Practical Research for Innovative Cancer Control from Japan Agency For Medical Research and development, AMED (20ck0106614h0001).

Authors' contributions

TM acquired the clinical data and drafted the manuscript. TM and AM reviewed all imaging data. TM, AM, SF, AN and IY were responsible for interpretation of the data and critical revision of the manuscript. TM and AM designed the study. All authors read and approved the final manuscript. 
Acknowledgements

We appreciate the help of Drs. Masanori Hisaoka and Kosuke Makihara, who diagnosed patients and obtained photographs of resected specimens and histological structure.

\section{References}

1. Ugai K, Sato S, Matsumoto K, Matsubara T, Mizuno K, Hirohata K. A clinicopathologic study of bony spurs on the pes anserinus. Clin Orthop Relat Res. 1988(231):130-4.

2. Fraser RK, Nattrass GR, Chow CW, Cole WG. Pes anserinus syndrome due to solitary tibial spurs and osteochondromas. J Pediatr Orthop. 1996;16(2):247-8.

3. Caffey J. Distal end of tibia and fibula. Pediatric X-ray diagnosis : a textbook for students and practitioners of pediatrics, surgery \& radiology 4th ed: Year Book Medical Publishers; 1961. p. 823.

4. Tiwari V, Sampath Kumar V, Poudel RR, Kumar A, Khan SA. Pes Anserinus Bursitis due to Tibial Spurs in Children. Cureus. 2017;9(7):e1427.

5. Keats T, Anderson M. Atlas of normal roentgen variants that may simulate disease, 9th ed. 9th ed: ELSEVIER; 2013. p. 590. figure 7-246.

6. Unni K, Inwards C. Osteochondroma. Dahlin's Bone Tumors, 6th ed: Lippincott Williams \& Wilkins 2009. p. 9-21.

7. Aiba H, Yamada S, Yamamoto N, Hayashi K, Miwa S, Tsuchiya H, et al. Spontaneous shrinkage of solitary osteochondromas. Skeletal Radiol. 2018;47(1):61-8.

8. Yasuda N, Nakai S, Nakai T, Outani H, Takenaka S, Hamada KI, et al. A pseudoaneurysm of the popliteal artery probably pierced by a bone spike arising in the spontaneously regressed osteochondroma: A case report. J Orthop Sci. 2018.

9. Zuntini M, Pedrini E, Parra A, Sgariglia F, Gentile FV, Pandolfi M, et al. Genetic models of osteochondroma onset and neoplastic progression: evidence for mechanisms alternative to EXT genes inactivation. Oncogene. 2010;29(26):3827-34.

10. Benjamin M, Toumi H, Ralphs JR, Bydder G, Best TM, Milz S. Where tendons and ligaments meet bone: attachment sites ('entheses') in relation to exercise and/or mechanical load. J Anat. 2006;208(4):471-90.

\section{Tables}


Table 1. Demographic and clinical characteristics of PABS cases and patients' radiologic and pathologic findings.

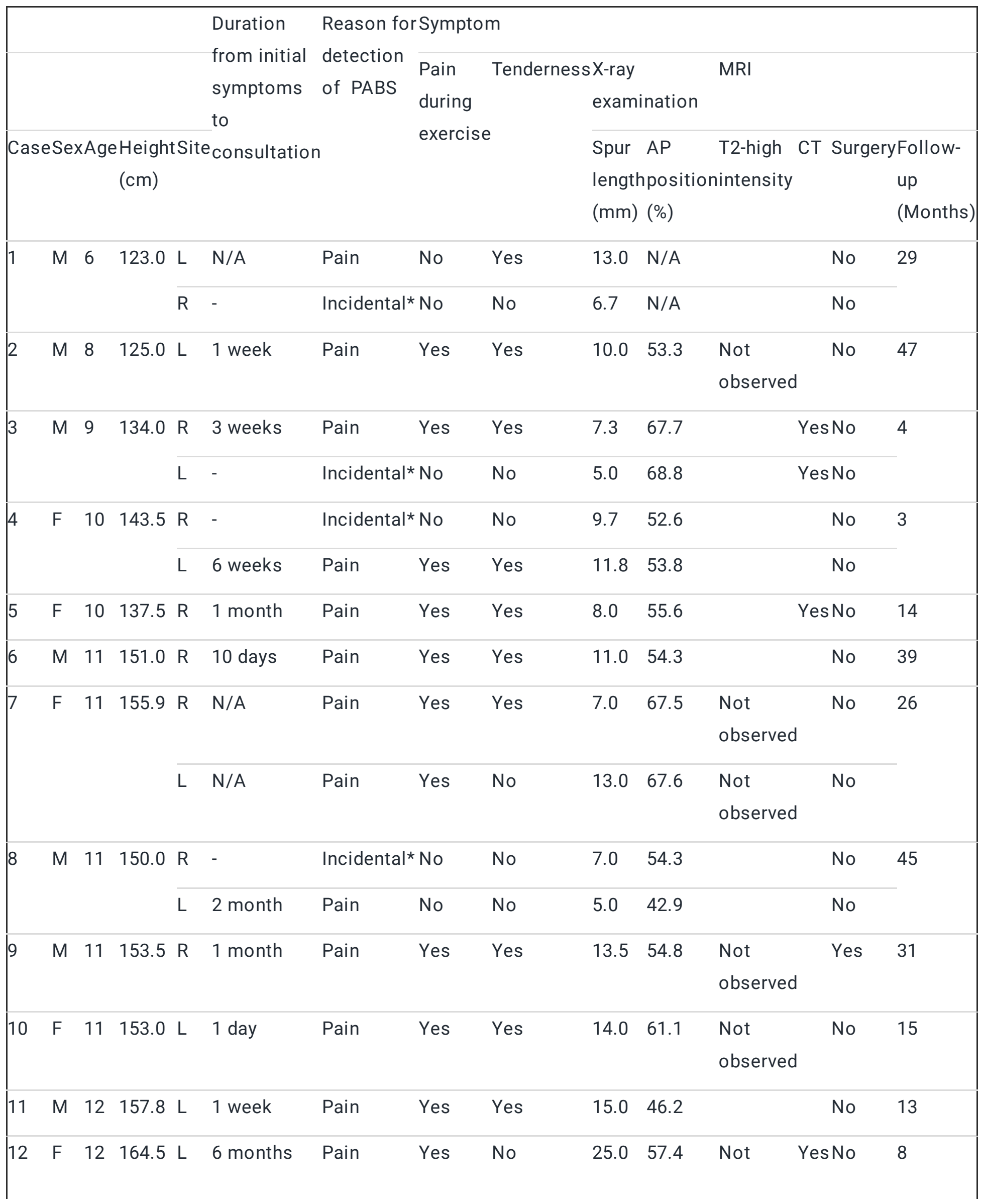




\begin{tabular}{|c|c|c|c|c|c|c|c|c|c|c|c|c|c|}
\hline & & & & & & & & & & & obs & & \\
\hline & & & & $\mathrm{R}$ & - & Incide & |* No & No & 10.0 & 59.4 & Not & YesNo & \\
\hline & & & & & & & & & & & obse & & \\
\hline 13 & $\mathrm{~F}$ & 12 & 158.9 & $\mathrm{R}$ & $\mathrm{N} / \mathrm{A}$ & Incide & I\#No & No & 5.0 & 72.1 & & YesNo & 29 \\
\hline 14 & $M$ & 13 & 163.3 & $\mathrm{~L}$ & 2 weeks & Pain & Yes & Yes & 21.0 & 51.2 & & YesNo & 12 \\
\hline 15 & $\mathrm{~F}$ & 13 & 161.0 & $\mathrm{R}$ & 2 weeks & Pain & Yes & Yes & 10.0 & 59.5 & & Yes No & 13 \\
\hline 16 & $\mathrm{~F}$ & 13 & 155.0 & $\mathrm{R}$ & 1 year & Pain & Yes & Yes & 9.0 & 42.9 & $\begin{array}{l}\text { Not } \\
\text { obse }\end{array}$ & No & 4 \\
\hline 17 & $M$ & 13 & 172.9 & $\mathrm{R}$ & 2 months & Pain & Yes & Yes & 6.0 & 67.3 & $\begin{array}{l}\text { Not } \\
\text { obse }\end{array}$ & $\begin{array}{l}\text { YesYes } \\
\text { d }\end{array}$ & 17 \\
\hline 18 & $\mathrm{M}$ & 14 & 176.0 & $\mathrm{R}$ & $\mathrm{N} / \mathrm{A}$ & Pain & No & No & 14.0 & 56.5 & & Yes No & 8 \\
\hline & & & & L & $\mathrm{N} / \mathrm{A}$ & Pain & No & No & 21.0 & 60.0 & & YesNo & \\
\hline 19 & $M$ & 14 & 168.5 & $\mathrm{R}$ & 6 months & Pain & Yes & Yes & 6.0 & 71.4 & $\begin{array}{l}\text { Not } \\
\text { obse }\end{array}$ & No & 18 \\
\hline 20 & $M$ & 15 & 166.9 & $\mathrm{~L}$ & 9 months & Pain & Yes & Yes & 5.0 & 72.9 & & No & 33 \\
\hline 21 & $\mathrm{M}$ & 16 & 164.0 & $\mathrm{~L}$ & 1 year & Pain & Yes & Yes & 8.0 & 68.0 & $\begin{array}{l}\text { Not } \\
\text { obse }\end{array}$ & No & 13 \\
\hline $\begin{array}{l}\text { L; I } \\
\text { the } \\
\text { gro } \\
\text { Inc } \\
\text { ide }\end{array}$ & ibia & $\begin{array}{l}\text { n/ } \\
\text { to } \\
\text { plate } \\
\text { al*; } \\
\text { d or }\end{array}$ & $\begin{array}{l}\text { A; not } \\
\text { the bas } \\
\text { and le } \\
\text { PABSs } \\
\text { X-ray }\end{array}$ & $\begin{array}{l}\text { ava } \\
\text { e o } \\
\text { vel } \\
\text { we } \\
\text { eva }\end{array}$ & $\begin{array}{l}\text { lable. Defin } \\
\text { the PABS } \\
\text { with the bas } \\
\text { e identified } \\
\text { luation of ar }\end{array}$ & $\begin{array}{l}\text { tion of } \\
\text { as divic } \\
\text { e of the } \\
\text { n X-ray } \\
\text { ankle }\end{array}$ & $\begin{array}{l}\text { rolate } \\
\text { by the } \\
\text { ur (see } \\
\text { btaine } \\
\text { in. Dia }\end{array}$ & $\begin{array}{l}(\mathrm{AP}) \\
\text { teropc } \\
\text { gure } 1 \\
\text { or con } \\
\text { nal lin }\end{array}$ & $\begin{array}{l}\text { on : Th } \\
\text { or diam } \\
\text { eral co } \\
\text { dicate }\end{array}$ & $\begin{array}{l}\text { e dista } \\
\text { heter } \mathrm{p} \\
\text { mpari } \\
\text { that ex }\end{array}$ & $\begin{array}{l}\text { ce fro } \\
\text { allel } t \\
\text { n. Inc } \\
\text { ninati }\end{array}$ & $\begin{array}{l}\text { he anterio } \\
\text { e proxim } \\
\text { tal\#; a Pf } \\
\text { were not }\end{array}$ & $\begin{array}{l}\text { edge of } \\
\text { tibial } \\
\text { S was } \\
\text { erformed. }\end{array}$ \\
\hline
\end{tabular}


Table 2. Literature review. N/A; not available, U; unilateral, B; bilateral.

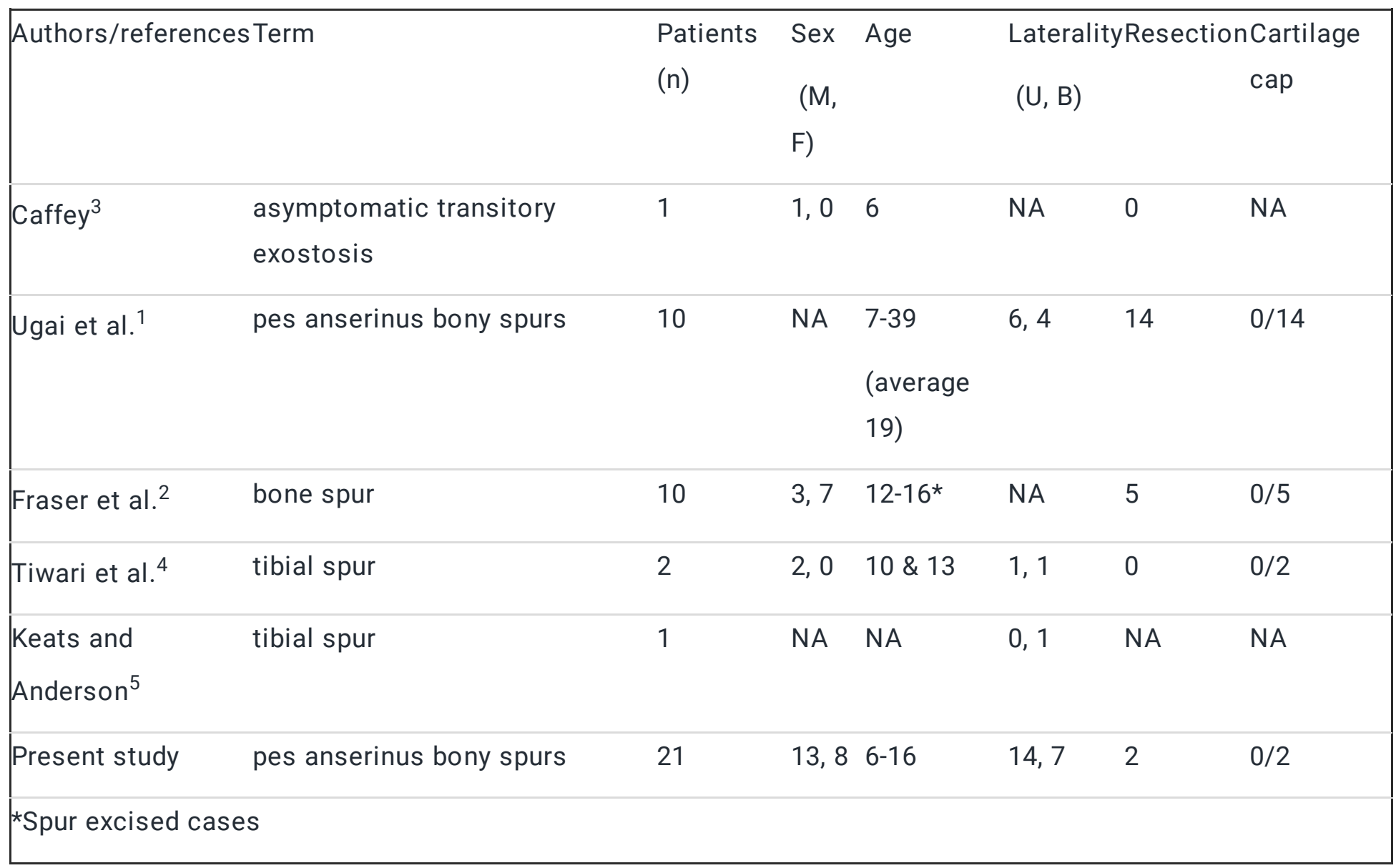

Figures 

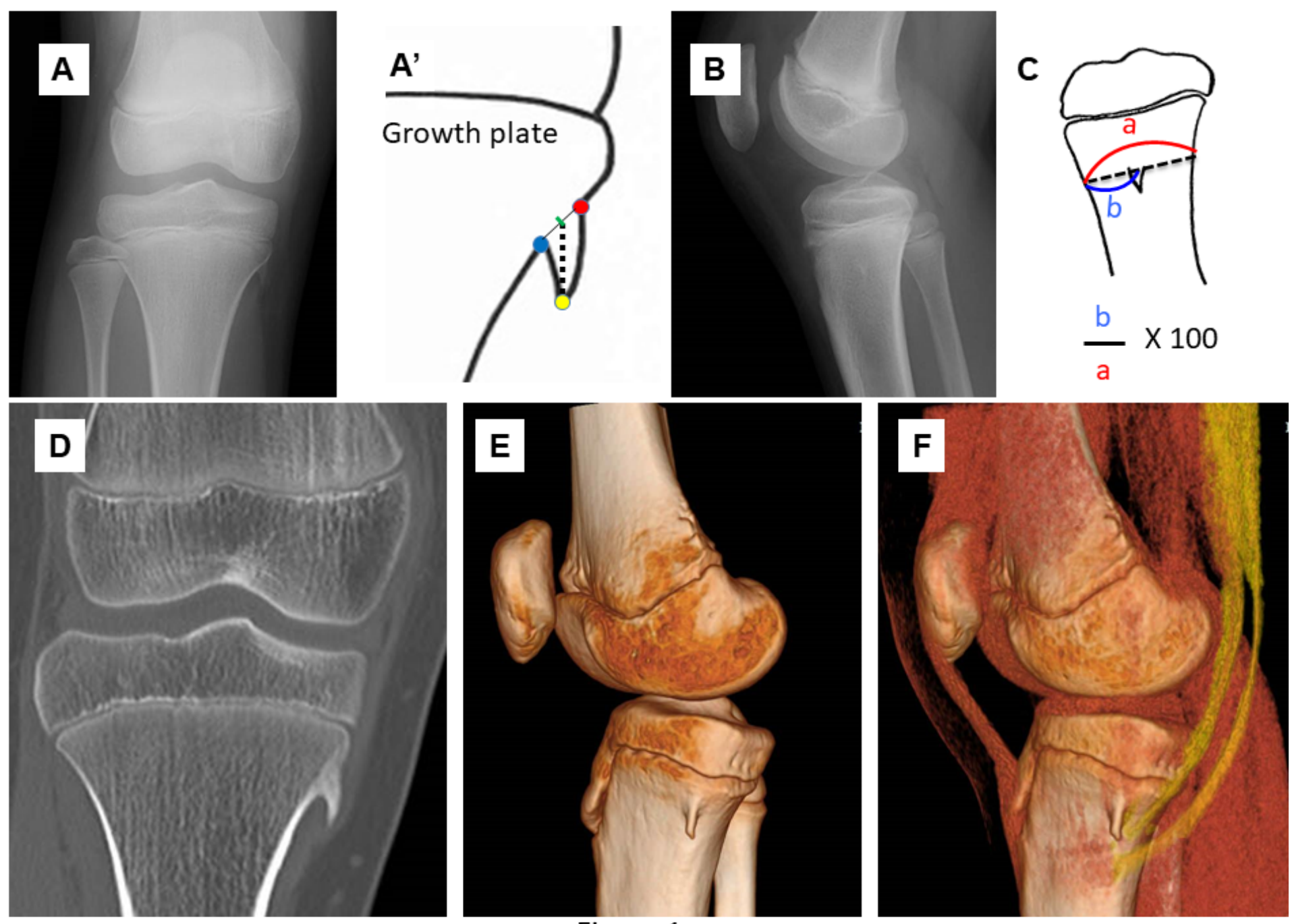

Figure 1

\section{Figure 1}

A patient with a pes anserinus bony spur on the right tibia (case \#5). A, B: AP (A) and lateral views (B) on plain $X$-ray show an icicle-like bony spur on the right proximal tibia. ( $A^{\prime}$ ) Measurement of the length of PABSs. The length of PABSs (dot-line) was determined by measuring the distance from the tip of PABSs (yellow circle) to the midpoint of the base of PABSs (green bar) on an anteroposterior plain X-ray. The midpoint of the base of PABSs was defined as center of a line to connect the most proximal point of the contour of PABSs (red circle) to the most distal point of that (blue circle). C: Calculation of the AP position of the spur in relation to the AP length of the tibia (a) and the length from the anterior edge to the base of the spur (b). D: Coronal reconstruction $C T$ image. $E, F$; 3D reconstruction of the knee without $(E)$ and with $(F)$ volume-rendered imaging of the soft tissue. Pes anserinus was colored with yellow. 
A

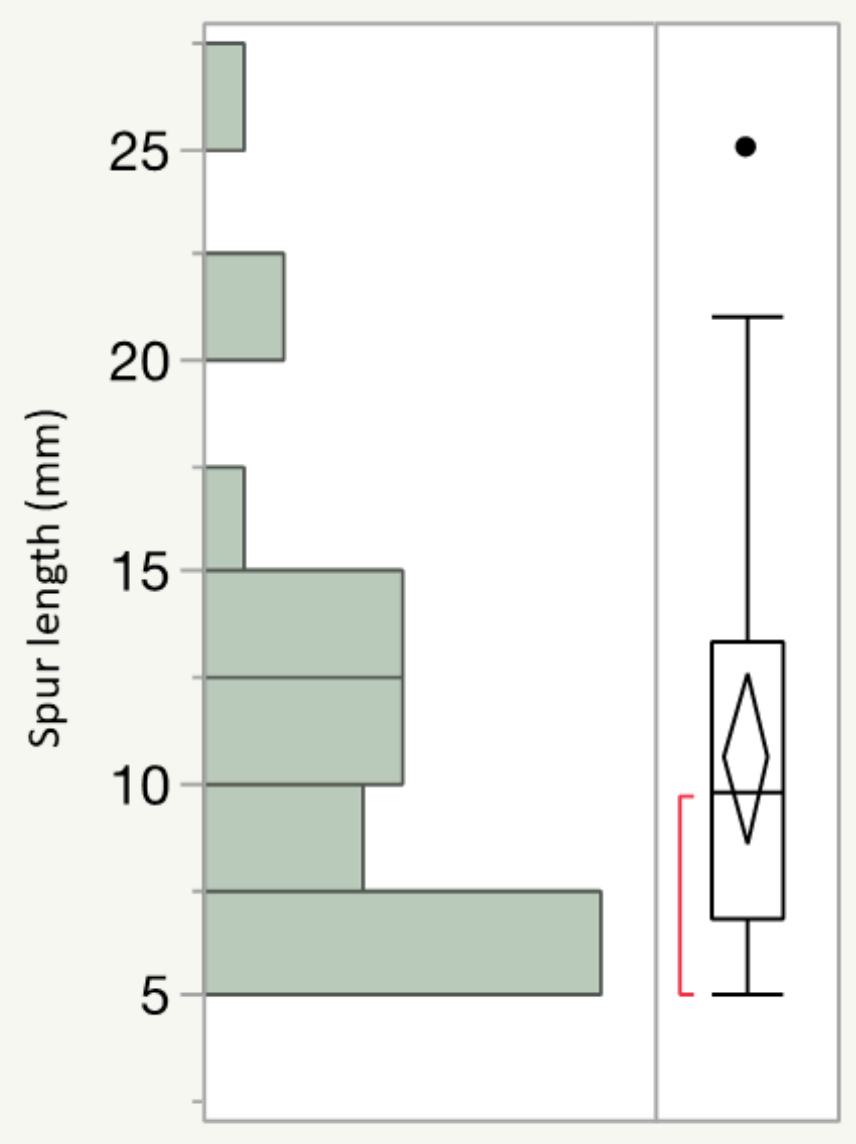

B

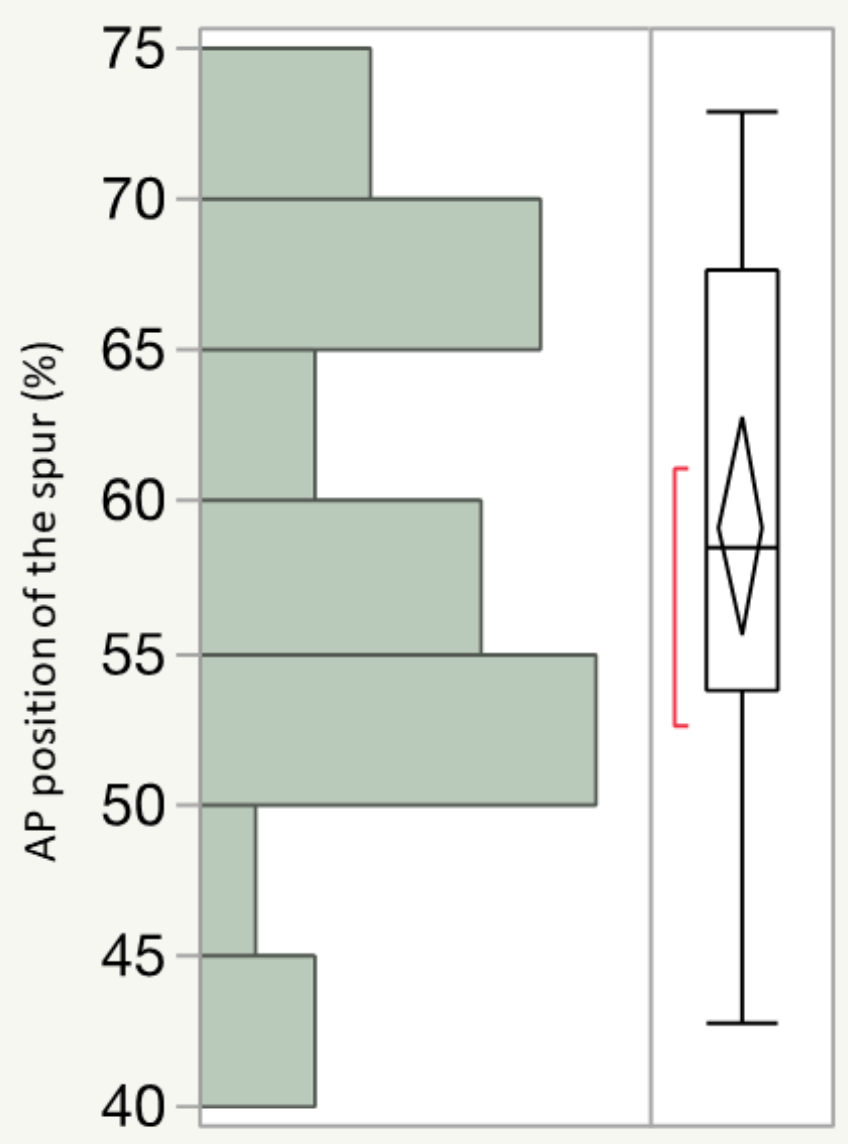

Figure 2

Figure 2

A and B: The distribution of the spur length on AP plain X-rays and the position of each PABS on lateral plain Xrays, respectively. 


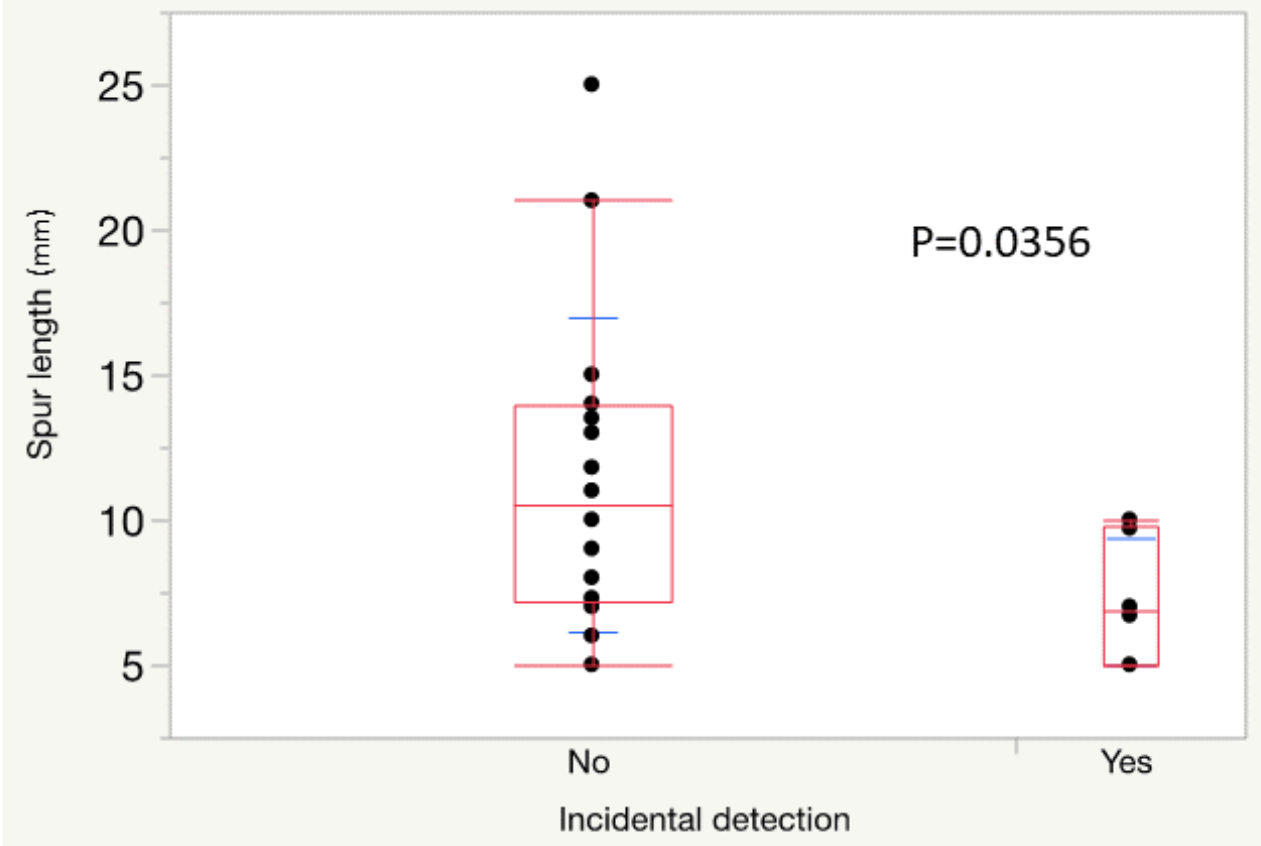

Figure 3

Figure 3

Correlation between spur length and patient characteristics. There was a significant difference between PABS length in the symptomatic detection group (No) and that in the incidental detection group (Yes). 

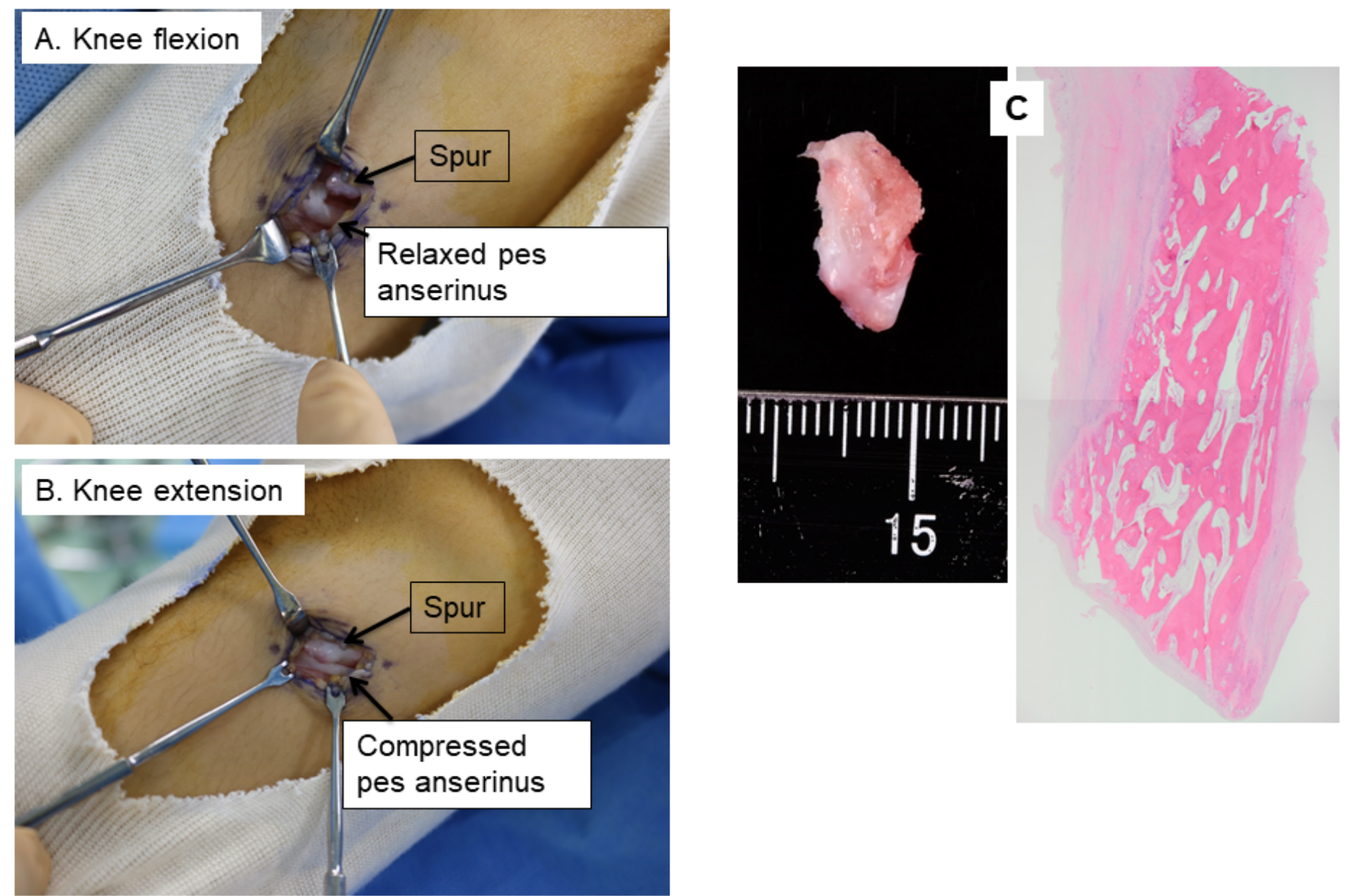

Figure 4

\section{Figure 4}

Intraoperative photographs (case \#9). A: The pes anserinus was relaxed and a gap was observed between the spur and the pes anserinus in knee flexion. B: The pes anserinus was compressed by the PABS at full knee extension. C. Photograph of a resected specimen (left). Histologically, the spur consists of lamellar bone trabeculae and fatty marrow, and no hyaline cartilaginous cap is seen in an haematoxylin-eosin-stained section (right). 


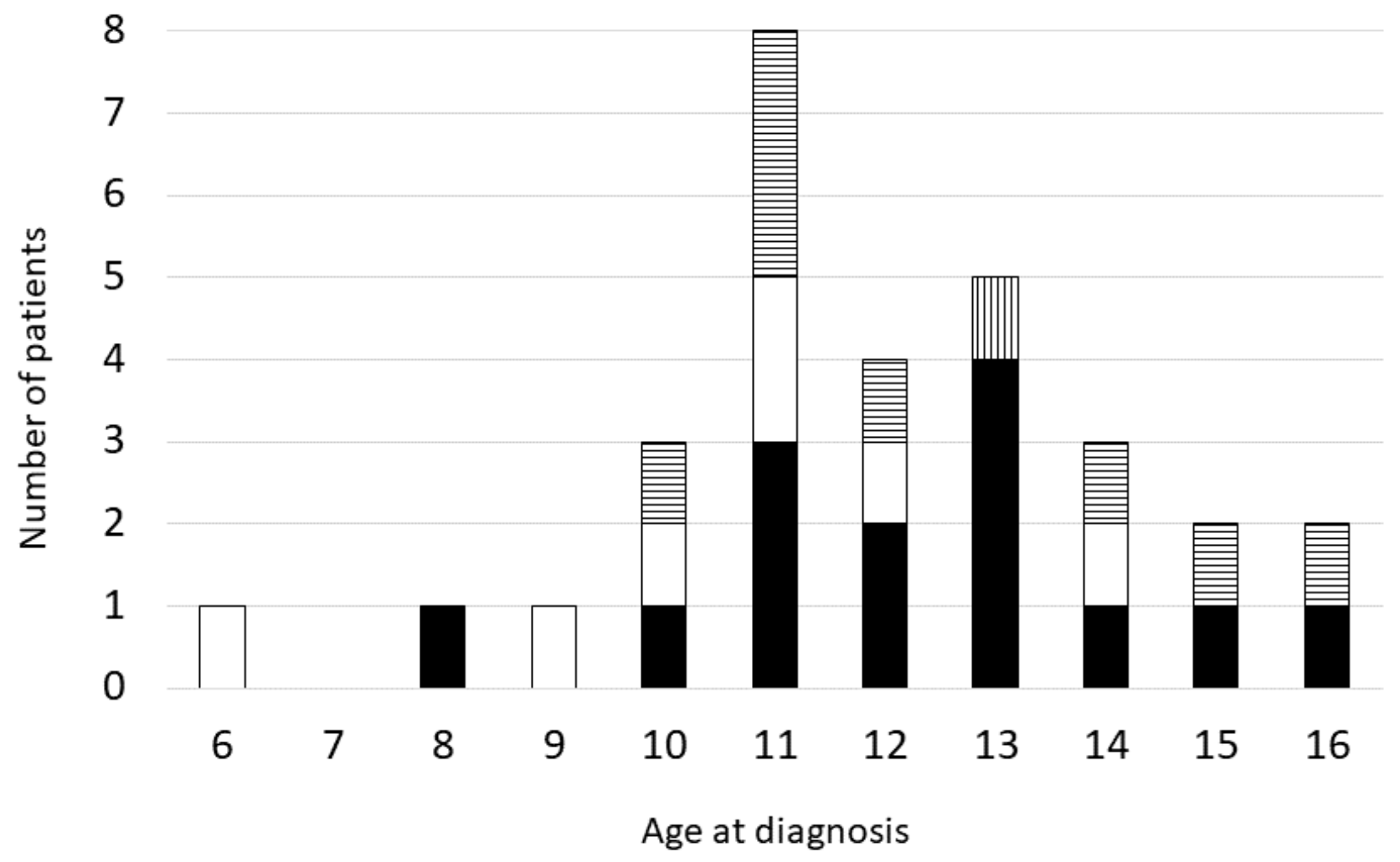

Figure 5

Figure 5

Number of patients with PABSs. The black and white bars indicate patients with unilateral and bilateral PABSs diagnosed by the orthopaedic oncology service, respectively (presented in Table 1). The horizontal striped bars and the vertical striped bars indicate newly identified patients with unilateral and bilateral PABSs, respectively. 